\title{
A SIMPLE PROCEDURE TO APPROXIMATE SLIP DISPLACEMENT OF FREESTANDING RIGID BODY SUBJECTED TO EARTHQUAKE MOTIONS Tomoyo TANIGUCHI ${ }^{1}$ and Takuya MIWA ${ }^{2}$
}

${ }^{1}$ Associate Professor, Department of Civil Engineering, Tottori University. 4-101 Koyama-Minami Tottori, 6808552, Japan. Tel +81-857-31-5287, Fax+81-857-28-7899, Email: t_tomoyo@cv.tottori-u.ac.jp

${ }^{2}$ Engineer, Bridge Design Department, TTK Corporation. 1020 Ooaza-Simotakai, Toride, 302-0038, Japan. Tel +81-297-78-1119, Fax +81-0297-78-5344, Email: Takuya_Miwa@ttk-corp.co.jp

(Former graduate student, Department of Civil Engineering., Tottori University)

\section{SUMMARY}

A simple calculation procedure for estimating absolute maximum slip displacement of a freestanding rigid body placed on the ground or floor of linear/nonlinear multi-story building during an earthquake is developed. The proposed procedure uses the displacement induced by the horizontal sinusoidal acceleration to approximate the absolute maximum slip displacement, i.e., the basic slip displacement. The amplitude of this horizontal sinusoidal acceleration is identical to either the peak horizontal ground acceleration or peak horizontal floor response acceleration. Its period meets the predominant period of the horizontal acceleration employed. The effects of vertical acceleration are considered to reduce the friction force monotonously. The root mean square value of the vertical acceleration at the peak horizontal acceleration is used. A mathematical solution of the basic slip displacement is presented. Employing over one hundred accelerograms, the absolute maximum slip displacements are computed and compared with the corresponding basic slip displacements. Their discrepancies are modeled by the logarithmic normal distribution regardless of the analytical conditions. The modification factor to the basic slip displacement is quantified based on the probability of the nonexceedence of a certain threshold. Therefore, the product of the modification factor and the basic slip displacement gives the design slip displacement of the body as the maximum expected value. Since the place of the body and linear/nonlinear state of building make the modification factor slightly vary, ensuring it to suit the problem is essential to secure prediction accuracy.

Keywords; horizontal sinusoidal acceleration, maximum expected slip displacement, coincident vertical acceleration, predominant period, peak horizontal ground acceleration, peak horizontal floor response acceleration.

\section{INTRODUCTION}

In recent highly developed and complicated social system the damage of structures no longer represents total effects of earthquakes. The functional loss of the social system as a result of the damage of nonstructural component should be taken into account. An appropriate design for nonstructural components to maintain minimum function in the event of earthquakes prevents from such systematic disorder. The former investigators clearly pointed out a need for investigation into seismic behavior of nonstructural components in order to assess 
their vulnerability [1]. An important class of nonstructural components, such as mechanical/electrical equipment, which is essentially modeled as a rigid body, is of interest to this study.

The effects of base excitation on a freestanding rigid body have been investigated by many researchers. They clarified five modes of the body (rest, slide, rock, slid-rock and jump), equations of motion and relation of wave properties of base excitation to these modes [2-6]. The senior author pointed out that the period of horizontal base excitation makes an important contribution to elongation of the slip displacement of the body in addition to the friction coefficient and peak horizontal acceleration [7].

Shao and Tung [8] prepared a chart which enabled to determine the mean-plus-standard deviation of the maximum sliding distance of an unanchored body. However, the study does not consider vertical excitation and its applicability to the body placed on the building floor. Including the vertical excitation, Lopez Garcia and Soong [1] presented the fragility information for sliding related failure modes. They classified the fragility curves according to the friction coefficient and peak horizontal ground acceleration. Although it gives the appropriate slip displacement if the body is on the ground, its prediction accuracy deteriorates when the body is on the building floor. Historically, Newmark [9] presented a simple formula to determine the sliding distance of a freestanding body subjected to a single rectangular acceleration pulse at the base concerning earthquake response of embankments. Choi and Tung [10] concluded that Newmark's formula could be used if an adjustment factor consisting of the friction coefficient and peak horizontal base acceleration was applied. However, the study is limited to the action of horizontal base excitation. In addition to that, prediction accuracy deteriorates if the body is on the building floor with a long natural period.

As mentioned above, the previous procedures for estimating the slip displacement of the body do not consider the period of horizontal excitation. Consequently, the prediction accuracy of the slip displacement of the body on the building floor may deteriorate because filtering effects of structure enhance the advent of a certain wave component in the floor response. This suggests the necessity of considering the period of horizontal excitation to approximate the slip displacement of the body set on the building floor.

An objective of this study is to develop a simple procedure that can approximate the absolute maximum slip displacement of the body during the earthquake wherever it is set on, i.e., the design slip displacement. In order to improve the deficiencies in the previous research, this study introduces the use of the single horizontal sinusoidal acceleration. Its amplitude is identical to either the peak horizontal ground acceleration or the peak horizontal floor response acceleration. Its period meets the predominant period of the horizontal acceleration employed. Generally, the peak horizontal ground acceleration is not always induced by the wave component with the predominant period of earthquake. On the other hand, due to filtering effects of structure, the peak horizontal floor response acceleration tends to be induced by the wave component with the predominant period of floor response. This is the advantage of the use of the horizontal sinusoidal acceleration.

The slip displacement of the body induced by the horizontal sinusoidal acceleration, i.e., the basic slip displacement, is used as the first approximation to the absolute maximum slip displacement of the body, since it 
gives an upper bound of the slip displacement under the single action of horizontal base acceleration. The discrepancy between the basic slip displacement and absolute maximum slip displacement are compiled to find the modification factor for the basic slip displacement. Since it is statistically examined based on the probability of nonexceedence of a certain threshold, the design slip displacement is given as the maximum expected value.

In contrast, Shao and Tung [8] suggested that the effects of the vertical ground motion on the mean-plusstandard deviation of the maximum sliding distance was small but it was indispensable to compute the slip displacement of the body accurately. In view of developing a simple calculation procedure and having a safety margin in slip displacement, this study introduces a monotonous reduction in friction force as if the uniform downward acceleration lasts while the body slips under action of the horizontal sinusoidal acceleration. The use of the root mean square value of the vertical acceleration at the peak horizontal acceleration [11, 12] is proposed as the uniform downward acceleration.

The fist part of the paper briefly describes the problem and the second part defines the horizontal sinusoidal acceleration and derives a mathematical solution of the basic slip displacement. The third and forth part examines the modification factor for the basic slip displacement of the body set on the ground and the building floor respectively. Three buildings with different stories and spans and liner/nonlinear state are considered.

\section{DESCRIPTION OF THE PROBLEM}

An equation of motion that governs the slip behavior of the body subjected to simultaneous horizontal and vertical acceleration is given as follows. Figure 1(a) and Figure 1(b) shows a mechanical model of the body placed on the ground and set on the building floor respectively. If we replace the shaking motion according to the problem, the subjects are essentially the same.
i) while slip
$\ddot{x}=-\ddot{z}_{h}-v\left(g+\ddot{z}_{v}\right) \operatorname{sign}(\dot{x})$
ii) while stationary
$\ddot{x}=0$
iii) slip commencement condition
$\left|\ddot{z}_{h}\right|>\mu\left(g+\ddot{z}_{v}\right)$
$\dot{x}=0$
iv) slip termination condition

where $\ddot{z}_{h}$ and $\ddot{z}_{v}$ are a pair of horizontal and vertical acceleration of either the ground or building floor, respectively. $g, \mu$ and $v$ are gravitational acceleration, static and kinetic friction coefficients, respectively. In order to simplify the problem, the static and kinetic friction coefficients are assumed to be the same and denoted as $\mu$ thereafter. The function $\operatorname{sign}(\dot{x})$ gives the sign of variable.

\section{BASIC SLIP DISPLACEMENT OF THE BODY}

\section{Simplification of the problem}

A simple treatment of the vertical acceleration is necessary to mathematically obtain the slip displacement of the body induced by the horizontal sinusoidal acceleration, i.e., the basic slip displacement of the body. Employing Eqs. (1) to (4) and all accelerograms listed on Table 1, the absolute maximum slip displacement of the body with/without the vertical ground acceleration is numerically computed. Each pair of horizontal and vertical accelerograms is scaled to $9 \mathrm{~m} / \mathrm{s}^{2}$ in the peak horizontal ground acceleration (PHGA). The friction coefficient is 
assumed to be 0.3 to 0.5 . The abscissa of Figure 1(c) is the ratio of the slip displacement induced by the simultaneous horizontal and vertical ground acceleration to the slip displacement induced by horizontal ground acceleration. The ordinate is the probability density of the frequency. Although the vertical ground acceleration does not always increase the slip displacement, it is better to ensure an adequate safety margin against the slip displacement. Since the slip motion of the body discontinuously occurs during the earthquake and lasts a short time, the effects of varying vertical acceleration on the slip motion of the body have few contributions. Therefore, this study introduces a monotonous reduction in friction force while the body slips [13]. To calculate the basic slip displacement of the body, Eqs. (1) and (3) are rewritten as follows.

$$
\begin{gathered}
\ddot{x}=-\ddot{z}_{h}-\mu(g-\sigma \cdot P V G A \cdot \eta) \operatorname{sign}(\dot{x}) \\
\left|\ddot{z}_{h}\right|>\mu(g-\sigma \cdot P V G A \cdot \eta)
\end{gathered}
$$

where $\ddot{z}_{h}$ is the horizontal sinusoidal acceleration defined by Eq. (7). $\sigma$ is the standard deviation of the ratio of the vertical ground acceleration to the peak vertical ground acceleration (PVGA) at the instant of PHGA. The senior author(s) compiled this ratio with 144 accelerograms. The probability density of this ratio was modeled by the normal distribution and the product of $\sigma$ and PVGA gave the root mean square value of the vertical ground acceleration. A pair of this vertical ground acceleration and PHGA was used for verification of the onset of slip of the unanchored flat-bottom cylindrical shell tank [11]. In addition, the probability density of this ratio computed with the ground acceleration was almost the same as that computed with the building floor response acceleration [12]. Therefore, this paper uses 0.46 as the value of $\sigma$ irrespective of the location of the body placed on. In case of the body set on the ground, this paper uses PVGA of each vertical accelerogram. In actual case scenario, although the value of PVGA is usually not provided by design codes, it can be determined by the ratio of PVGA to PHGA [14] or the well-known empirical rule PVGA $=0.5$ or 0.66 PHGA. In contrast, $\eta$ is the magnification factor for the vertical floor response given by the quotient of the peak vertical floor response acceleration (VFRA) by PVGA. This paper uses the peak VFRA of each result of time history analysis as the value of $P V G A \cdot \eta$. In actual case scenario, $\eta$ is determined by the response amplitude. Therefore, $\eta$ is 1.0 if the body is set on the ground.

\section{Horizontal sinusoidal acceleration}

The amplitude of the horizontal sinusoidal acceleration, $A_{g x} g$, is identical to PHGA if the body is placed on the ground. In contrast, it should be identical to the peak horizontal floor response acceleration (HFRA) if the body is set on the building floor.

$$
\ddot{z}_{h}=A_{g x} g \sin \left(\frac{2 \pi}{T} t\right)
$$

where $T$ is the predominant period of the horizontal acceleration (PPHA) of either the horizontal ground acceleration or floor response acceleration. In case of the body set on the ground, this paper uses PPHA determined by the period which maximizes a pseudo-acceleration spectrum of each horizontal accelerogram. In actual case scenario, PPHA is determined by the natural period of soil, since it will be predominant over other 
wave period in the earthquake. Ref. [14] gives the calculation of the natural period of soil. In case of the body set on building floor, this paper uses PPHA determined by FFT analysis of each horizontal floor acceleration. For the convenience of actual case scenario, the calculation of the predominant period of the horizontal floor response acceleration is proposed latter.

\section{Mathematical solution}

Solve Eqs. (5) to (7) mathematically to obtain the basic slip displacement of the body. Firstly, assume an absence of the vertical acceleration to simplify the problem. Figures 1(d) and 1(e) depict the typical time history of slip motion of the body subjected to the horizontal sinusoidal acceleration with $A_{g x} g=7 \mathrm{~m} / \mathrm{s}^{2}, T=6.28 \mathrm{~s}$ and $\mu=0.4$. When the body undergoes the horizontal base acceleration, the body begins to slip when the horizontal inertia force overcomes the friction force. From Eqs. (6) and (7), the time, $t_{0}$, for the onset of slip is calculated as:

$$
t_{0}=\frac{T}{2 \pi} \operatorname{Sin}^{-1} \frac{\mu}{A_{g x}}
$$

Despite the time history of horizontal base acceleration the slip acceleration of the body is uniform. The velocity of the body at arbitrary time is calculated as:

$$
\dot{x}=\mu g t+C_{1}
$$

Similarly, the velocity of the base is calculated as:

$$
\dot{z}_{h}=-A_{g x} g \frac{T}{2 \pi} \cos \frac{2 \pi}{T} t
$$

where the initial velocity of the base is assumed to be $-A_{g x} g T / 2 \pi$ to derive Eq. (10). In contrast, $C_{1}$ in Eq. (9) is the constant of integration and should be determined as that the body has the same velocity of the base at the onset of slip.

$$
C_{1}=-\frac{T}{2 \pi}\left(\mu g \operatorname{Sin}^{-1} \frac{\mu}{A_{g x}}+A_{g x} g \cos \left(\operatorname{Sin}^{-1} \frac{\mu}{A_{g x}}\right)\right)
$$

From Fig. 1(e), the slip terminates when the velocity of the body and base become the same. The time is calculated by Eqs. (9), (10) and (11) as follows.

$$
\mu g t-\frac{T}{2 \pi}\left(\mu g \operatorname{Sin}^{-1} \frac{\mu}{A_{g x}}+A_{g x} g \cos \left(\operatorname{Sin}^{-1} \frac{\mu}{A_{g x}}\right)\right)=-A_{g x} g \frac{T}{2 \pi} \cos \frac{2 \pi}{T} t
$$

Solve Eq. (12) for $t$ to find the slip termination time, $t_{1}$. Since Eq. (12) is, however, the transcendental function in terms of the cosine function, this study tries to find its approximate solution employing the Taylor's series. From Figs. 1(d) and 1(e), since the slip terminates around $t=T / 2$, the Taylor's series of the cosine function around $t=T / 2$ is used.

$$
\cos \frac{2 \pi}{T} t \approx-1+\frac{2 \pi^{2}}{T^{2}}\left(t-\frac{T}{2}\right)^{2} \cdots
$$

Substitution of Eq. (13) into Eq. (12) yields the slip termination time, $t_{1}$, as follows. 


$$
t_{1}=\frac{T}{2 \pi A_{g x}}\left(-\varphi+\sqrt{\varphi^{2}+2 A_{g x}\left\{\phi+A_{g x}\left(1-\frac{\pi^{2}}{2}\right)\right\}}\right) \text {, where } \varphi=\mu-\pi A_{g x}, \phi=\mu \operatorname{Sin}^{-1} \frac{\mu}{A_{g x}}+A_{g x} \cos \left(\operatorname{Sin}^{-1} \frac{\mu}{A_{g x}}\right)
$$

Therefore, the basic slip displacement, $x_{h}^{\text {Sin }}$, is calculated by integrating the relative slip acceleration over the duration of the slip.

$$
x_{h}^{S i n}=\int_{t_{0}}^{t_{1}} \int_{t_{0}}^{t_{1}}\left(\mu g-A_{g x} g \sin \frac{2 \pi}{T} t\right) d t d t=\frac{1}{2} \mu g t_{1}^{2}+\frac{A_{g x} g T^{2}}{4 \pi^{2}} \sin \frac{2 \pi}{T} t_{1}+D_{1} t_{1}+D_{2}
$$

where $\quad D_{1}=-\mu g t_{0}-\frac{A_{g x} g T}{2 \pi} \cos \frac{2 \pi}{T} t_{0}, \quad D_{2}=\frac{1}{2} \mu g t_{0}^{2}-\frac{A_{g x} g T^{2}}{4 \pi^{2}} \sin \frac{2 \pi}{T} t_{0}+\frac{A_{g x} g T t_{0}}{2 \pi} \cos \frac{2 \pi}{T} t_{0}$

It is worth nothing that $t_{0}$ and $t_{1}$ are determined by only parameters that specify the slip motion of the body. Figures 2(a) to 2(j) show the slip motion of the body induced by the sinusoidal base acceleration. The title of each figure shows a combination of analytical condition $A_{g x}=0.5$ to $1.5, T=0.3$ to 1.0 and $\mu=0.3$ to 0.5 . Although the slip termination time varies $t=5 T / 8$ to $3 T / 4$ with the change in the analytical condition, Eq. (15) well approximates the exact slip displacement computed numerically (See Figure 2(k)). Since the value of the cosine function increases monotonously in the range considered herein, the Taylor's series of the cosine function around $t=T / 2$ favorably works.

Finally, consider the action of the monotonous downward vertical acceleration. Simply replacing the friction coefficient, $\mu$, of Eqs. (8) to (15) by the nominal friction coefficent $\mu^{\prime}=\mu(1-P V G A \cdot \eta \cdot \sigma / g)$, the basic slip displacement $x_{h, v}^{S i n}$ is calculated by the same manner.

\section{DESIGN SLIP DISPLACEMNT OF THE BODY ON THE GROUND}

\section{Methodologies}

Although the horizontal sinusoidal acceleration possesses representative characters of the earthquake wave, it does not include all properties that randomly appear in the earthquake wave. Therefore, the basic slip displacement is naturally different from the absolute maximum slip displacement induced by the earthquake. This paper considers it as the estimation error of the proposed procedure since the basic slip displacement is deterministically calculated while the absolute maximum slip displacement is influenced by the randomness of the earthquake wave. The discrepancies between them are compiled. The modification factor for the basic slip displacement to calculate the design slip displacement is statistically examined.

\section{Earthquake records}

This study uses 104 accelerograms observed around Japan [15]. The slip displacement discussed herein is inevitably affected by regional properties of accelerogram samples, although these earthquakes are of different characteristics in terms of energy content in various frequency bands, duration, and variation of intensity with respect to time (See Table 1). According to the classification of soil type on Ref. [14], the natural period of hard soil is less than $0.2 \mathrm{~s}$, that of soft soil is longer than $0.6 \mathrm{~s}$, and remaining is classified as the medium soil. Each soil type has 34,42 , and 28 accelerograms, respectively. The time history of horizontal acceleration is first 
normalized. That is, for each acclelerogram, PHGA is scaled to a value of $g$. When one is interested in the statistics of the slip displacement of a body to say, $0.4 \mathrm{~g}$, other than $g$, all horizontal acceleration time histories are scaled by multiplying them by the value of 0.4 . Maintaining the relation between PHGA and PVGA of each pair of accelerograms, the time history of vertical acceleration is also normalized and scaled in a same manner. Employing a pair of scaled accelerograms in $\ddot{z}_{h}$ and $\ddot{z}_{v}$, the time history of slip displacement is numerically computed by ACSL with 0.001 seconds intervals [16].

\section{Modification factor for basic slip displacement}

The absolute maximum slip displacement of the body induced by the earthquake wave, $\left|x_{h, v}^{E q}\right|_{M a x}$, is numerically computed and compared with the corresponding basic slip displacement, $x_{h, v}^{\operatorname{Sin}}$, induced by the horizontal sinusoidal acceleration with the nominal friction coefficient, $\mu^{\prime}$. Introducing the slip ratio, $\beta_{h, v}$, the estimation errors are statistically examined.

$$
\beta_{h, v}=\frac{\left|x_{h, v}^{E q}\right|_{\operatorname{Max}}}{x_{h, v}^{\operatorname{Sin}}}
$$

There are three primary reasons that the basic slip displacement is different from the absolute maximum slip displacement.

1) The wave form of earthquake is different from that of the horizontal sinusoidal acceleration while the body slips.

2) The wave period of earthquake is different from that of the horizontal sinusoidal acceleration while the body slips.

3) Since the wave of earthquake is naturally asymmetric, the slip displacement is sometimes one-sided.

Since these are general characteristics of estimation errors and interpreted accordingly, the modification factor for the basic displacement to approximate the absolute maximum slip displacement is statistically defined.

Figures 3(a) to 3(c) show the probability density of the slip ratio, $\beta_{h, v}$. The slip ratio is classified according to a combination of PHGA and $\mu$. The legend in each figure shows the value of $\mu$. PHGA and friction coefficient are assumed to be $5 \mathrm{~m} / \mathrm{s}^{2}$ to $9 \mathrm{~m} / \mathrm{s}^{2}$ and 0.3 to 0.5 respectively. From these figures, the slip ratio, $\beta_{h, v}$, possesses almost the same probability density irrespective of the combination of PHGA and $\mu$. Figure 3(d) shows the probability density of the slip ratio, $\beta_{h, v}$, classified according to the soil type without identification of PHGA and $\mu$. The slip ratio, $\beta_{h, v}$, also possesses almost the same probability density irrespective of soil types. Consequently, the probability density of the slip ratio, $\beta_{h, v}$, is compiled irrespective of analytical conditions. Figure 3(e) shows the probability density of the slip ratio, $\beta_{h, v}$, of all results. The distribution is modeled by the logarithmic normal distribution function whose mean and standard deviation are 0.83 and 0.78 respectively. The solid line in Figure 3(e) presents an approximation. Figure 4 shows the probability of the slip ratio $\beta_{h, v}$ converted from Figure 3(e). A table below the figure shows the value of the modification factor, $\beta_{h, v}^{\text {Prob }}$, for the selected probability of the nonexceedence of the specified threshold. The product of the modification factor, 
$\beta_{h, v}^{\text {Prob }}$, and the basic slip displacement, $x_{h, v}^{\text {Sin }}$, gives the design slip displacement, $x_{h, v}^{E x p}$, as the maximum expected value.

$$
x_{h, v}^{E x p}=\beta_{h, v}^{\operatorname{Pr} o b} \cdot x_{h, v}^{\operatorname{Sin}}
$$

Here, in the absence of vertical ground motion, our previous study revealed that the mean and standard deviation of the slip ratio were 1.03 and 0.71 , respectively [13]. Having the value of 1.03 in the mean suggests that the largest slip displacement is likely induced by the wave with PPHA and corroborates the importance of consideration of PPHA. However, the monotonous reduction in friction force slightly overestimates the effects of the vertical ground acceleration on the slip displacement of the body as a consequence of that the inclusion of vertical ground motion decreases the mean 19\%. In contrast, since these standard deviations are the same order, the proposed procedure maintains the estimation accuracy if the vertical ground acceleration is considered.

\section{DESIGN SLIP DISPLACEMNT OF THE BODY ON THE BUILDING FLOOR}

\section{Methodologies}

The following section tries to apply the proposed procedure to estimate the slip displacement of the body set on the building floor. All parameters that define the horizontal sinusoidal acceleration, i.e., PHGA, PVGA and PPHA, should be replaced by the peak HFRA, peak VFRA and corresponding PPHA, respectively. The absolute maximum slip displacement of the body induced by the floor response acceleration is compapred with the corresponding basic slip displacement. The modification factor for the basic slip displacement is statistically examined.

\section{Time history of floor response}

This investigation uses three actual concrete building models with different stories and spans and 3\% structural damping illustrated in Figure 5(a). The dimensions of each column and beam are shown in Figure 5(b). Figure 5(c) shows the natural period of each mode of each building. PHGA of all accelerograms listed in Table 1 is scaled to 250gal for linear analysis and 500gal for nonlinear analysis. In nonlinear analysis, all columns maintain elasticity, while plastic hinges appear on beams at beam-column connections. The nonlinear property of the beam is modeled by Takeda-model [17]. The time history of HFRA and VFRA are picked up at top and middle floors of each building. The dynamic analysis of building is carried out by commercial software TDAP III [17] without a contribution to slip of the body. Since the nonlinear model of the beam affects the properties of floor response, the nature of slip behavior at other type of nonlinear model differs from that observed herein. Since it affects the statistical properties of the slip ratio, the other slip ratio is necessary for the case.

\section{Predominant period of horizontal floor response acceleration}

To know the predominant period of HFRA is essential to secure the prediction accuracy of the slip displacement of the body set on the building floor. Strictly speaking, we need to know the predominant period of HFRA that causes the largest slip displacement in the time history because it dominates the absolute maximum slip displacement of the body, i.e., dominant slip displacement. In most cases, this HFRA is identical to the peak HFRA. Therefore, picking up the HFRA for three seconds around the onset of the dominant slip displacement, 
the component of wave period is investigated by FFT analysis. To find typical characters in the predominant period of HFRA, the following investigation uses twenty accelerograms randomly chosen from Table 1. Figures 6(a) to 6(c) show the predominant period of HFRA observed at the specified floor of linear building while Figures 6(d) to 6(f) show that of nonlinear building. The abscissa of these figures shows the predominant period of earthquake input to the building model determined by a pseudo-acceleration spectrum of each earthquake. The ordinate of these figures shows the predominant period of HFRA that causes the dominant slip displacement. The horizontal lines in each figure show the natural period of the first three modes of each building. From these figures, the following typical characteristics are found [18].

1) The predominat period of HFRA is close to that of horizontal ground acceleration if the nonlinearity of structure appears before HFRA reaches its peak (See Group 1 in Figures 6(d) to 6(f)). Since the nonlinearity of structure diminishes filtering effects of structure, properties of earthquake wave appear directly on the floor response.

2) The predominant period of HFRA is not close to that of horizontal ground acceleration when the structure keeps elasticity or just reaches nonlinear region at an instant of the peak HFRA (See Figures 6(a) to 6(c) and Group 2 in Figures 6(d) to 6(f)). However, the predominant period of HFRA is not always identical to either the natural period of modes of structure, since the structure is always in transient response during an earhtquake. It implies the necessity of developing an estimation method of the predominant period of HFRA. This paper attemps to predict the predominant period of HFRA by superposing the natural period of each mode inspired from the root mean square method.

Figures $6(\mathrm{~g})$ to $6(\mathrm{i})$ examine the prediction accuracy of the predominant period of HFRA with all acclerograms, floor loction, building types and linear/nonlinear state of building. The proposed method can adequately predict the predominant period of HFRA despite the analytical conditions.

\section{Modification factor for basic slip displacement (buildings in linear state)}

Firstly, consider the buildings in linear state. The absolute maximum slip displacement of the body induced by

HFRA and VFRA, $\left|x_{h, v}^{F l}\right|_{\text {Max }}$, is numerically computed and compared with the corresponding basic slip displacement, $x_{h, v}^{S i n}$, induced by the horizontal sinusoidal acceleration with the nominal friction coefficient, $\mu^{\prime}$. Introducing the slip ratio, $\beta_{h, v}$, the estimation errors are statistically examined.

$$
\beta_{h, v}=\frac{\left|x_{h, v}^{F l}\right|_{\text {Max }}}{x_{h, v}^{\operatorname{Sin}}}
$$

Figures 7(a) to 7(f) show the probability density of the slip ratio, $\beta_{h, v}$, classified according to the floor location, building type and $\mu$. Similar to, $\beta_{h, v}$, observed on the ground, the slip ratio, $\beta_{h, v}$, forms a certain probability density despite the analytical conditions. Figure $7(\mathrm{~g})$ is the probability density of the slip ratio, $\beta_{h, v}$, of all results. The distribution is modeled by the logarithmic normal distribution function whose mean and standard deviation are 0.70 and 0.54 , respectively. The solid line in Figure $7(\mathrm{~g})$ presents an approximation. Figure 8 
shows the probability of the slip ratio, $\beta_{h, v}$, converted from Figure $7(\mathrm{~g})$. A table below the figure shows the value of modification factor, $\beta_{h, v}^{\text {Prob }}$, for the selected probability of the nonexceedance of the specified threshold. Employing Eq. (17), the design slip displacement, $x_{h, v}^{E x p}$, is calculated as the product of the modification factor, $\beta_{h, v}^{\text {Prob }}$, and the basic slip displacement, $x_{h, v}^{\text {Sin }}$. This simplification imposes restriction on the proposed method. For instance, adoption of the unique value of $\beta_{h, v}$ in estimating the slip displacement of the body set on the first floor where the filtering effects of structure cannot be expected leads considerable error.

A comparison of Figures 3(e) and 7(g) yields advantage of the use of the horizontal sinusoidal acceleration since the standard deviation of Figure $7(\mathrm{~g})$ is $69 \%$ of that of Figure 3(e). The inherent randomness in the earthquake is diminished by filtering effects of structure and the wave form of HFRA becomes similar to that of the horizontal sinusoidal acceleration. In contrast, in addition to overestimation of effects of the vertical ground acceleration, decrease in the mean is caused by the estimation error of the predominant period of HFRA because the proposed method tends to estimate the predominant period of HFRA to be slightly longer. Therefore, ensuring the modification factor to suit the problem is essential to secure the prediction accuracy of the slip displacement.

\section{Modification factor for basic slip displacement (buildings in nonlinear state)}

Secondly, consider the buildings in nonlinear state. Employing Eq. (18), the slip ratio, $\beta_{h, v}$, is statistically examined.

Figures 9(a) to 9(f) show the probability density of the slip ratio, $\beta_{h, v}$, classified according to the floor location, building type and $\mu$. Similar to previous discussion, the slip ratio $\beta_{h, v}$ forms a certain probability density despite the analytical conditions. Figure $9(\mathrm{~g})$ shows the probability density of the slip ratio $\beta_{h, v}$ of all results. The distribution is modeled by the logarithmic normal distribution function whose mean and standard deviation are 0.38 and 0.51 respectively. The solid line in Figure $9(\mathrm{~g})$ presents an approximation. Figure 10 shows the probability of the slip ratio, $\beta_{h, v}$, converted from Figure $9(\mathrm{~g})$. A table below the figure shows the value of modification factor, $\beta_{h, v}^{\text {Prob }}$, for the selected probability of the nonexceedance of the specified threshold. Employing Eq. (17), the design slip displacement, $x_{h, v}^{E x p}$, is calculated as the product of the modification factor, $\beta_{h, v}^{\text {Prob }}$, and the basic slip displacement, $x_{h, v}^{\text {Sin }}$.

From Figures $7(\mathrm{~g})$ and $9(\mathrm{~g})$, the prediction accuracy slightly deteriorates since the mean decreases $46 \%$ but the standard deviation maintains the same order. In addition to the overestimation of effects of the vertical ground acceleration, decrease in the mean is caused by the dissimilarity of the wave form between HFRA of the building in the nonlinear state and the horizontal sinusoidal acceleration [18]. Figure 11(a) shows the time history of HFRA and slip acceleration of the body observed at linear building. Figure 11(b) magnifies the onset of the dominant slip displacement. The approximation of the floor motion by the horizontal sinusoidal acceleration is also plotted. The similarity between HFRA and the horizontal sinusoidal acceleration and slip acceleration induced by them corroborates the applicability of the proposed procedure. In contrast, Figures 11(c) and 11(d) show those observed at nonlinear building. The dissimilarity between HFRA and the horizontal sinusoidal acceleration and its effects on the slip acceleration can be seen. The advantage of the use of the sinusoidal wave 
slightly diminishes. However, since these standard deviations still keep the same order, the proposed procedure maintains the estimation accuracy if the nonlinearity of the structure is considered. Therefore, ensuring an adequate modification factor to suit the problem is essential to secure the prediction accuracy of the slip displacement.

\section{CONCLUSION}

This paper proposes the use of the horizontal sinusoidal acceleration to approximate the slip displacement of the body wherever it is set on during an earthquake. A mathematical solution of the slip displacement of the body induced by the horizontal sinusoidal acceleration, i.e., the basic slip displacement, is presented in a practical form. The product of the basic slip displacement and the modification factor gives the design slip displacement as the maximum expected value. The modification factor is quantified as an estimation error between the basic

slip displacement and the absolute maximum slip displacement based on the probability of the nonexceedence of a certain threshold. It forms a certain probability density despite the acceleration intensity and the friction coefficient. However, the modification factors are slightly different according to the place of the body and linear/nonlinear state of building. In order to secure the prediction accuracy of the slip displacement of the body, ensuring the modification factor to suit the problem is essential.

\section{REFERENCES}

1. Lopez Garcia D, Soong TT. Sliding fragility of block-type non-structural components, Part I: unrestrained components, Earthquake Engineering and Structural Dynamics 2003; 32(1): 111-129.

2. Ishiyama Y. Motions of rigid bodies and criteria for overturning by earthquake excitations. Earthquake Engineering and Structural Dynamics 1982; 10: 635-650.

3. Shenton III HW, Jones NP. Base excitation of rigid bodies. I: formulation. Journal of Engineering Mechanics, ASCE, 1991; 117: 2286-2306.

4. Shenton HW III. Criteria for initiation of slide, rock, and slide-rock rigid-body modes. Journal of Engineering Mechanics, ASCE, 1996; 122(7): 690-693.

5. Taniguchi T and Murayama T. Study of free liftoff induced slip behavior of rectangular rigid bodies. PVP, Seismic Engineering, ASME, 2001: 428-1: 123-130.

6. Taniguchi T. Experimental and analytical study of free lift-off motion induced slip behavior of rectangular rigid bodies. Journal of Pressure Vessel Technologies, ASME, 2004: 126(1): 53-58.

7. Taniguchi T. Nonlinear response analyses of rectangular rigid bodies subjected to horizontal and vertical ground motion. Earthquake Engineering and Structural Dynamics, 2002; 31: 1481-1500.

8. Shao Y, Tung CC. Seismic response of unanchored bodies. Earthquake Spectra, 1999; 15(3): 523-536.

9. Newmark NM. Effects of earthquakes on dams and embankments, Geotechnique, 1965; 15(2): 139-160.

10. Choi B, Tung CC. Estimating sliding displacement of an unanchored body subjected to earthquake excitation. Earthquake Spectra, 2002; 18(4): 601-613.

11. Taniguchi T, Murayama T, Mentani Y, Komori H, Yoshihara T, Slip verification method for the flat- 
bottom cylindrical shell tank subjected to horizontal and vertical ground motion. Journal of Structural Mechanics and Earthquake Engineering, JSCE, 2000; 611(I-53): 95-105 (Japanese).

12. Taniguchi $\mathrm{T}$, Takeshita M. Coincident vertical response analysis of structure at maximum horizontal response. 13WCEE, No.433, 2004.

13. Taniguchi T, Miwa T. Slip displacement analysis of freestanding rigid bodies subjected to earthquake motions. 13WCEE, No.437, 2004.

14. Japan Road Association. Specifications for Highway Bridges-Part V: Seismic Design. 2002 (Japanese).

15. National Information Center for Earthquakes and Disasters, http://www.bosai.go.jp/

16. ACSL Reference Manual; Edition 11.1, MGA Software: Concord, 1995.

17. TDAP III ver.2.11.01. ARK Information Systems, 2002.

18. Miwa T. A study of slip displacement of rigid body considering period of shaking. Mater thesis, Tottori University, 2004 (Japanese) 
Tomoyo Taniguchi

Table 1 List of accelerograms

\begin{tabular}{|c|c|c|c|c|c|c|c|}
\hline Name of Earthquake & PHGA(gal) & PVGA(gal) & PPHA(s) & Name of Earthquake & PHGA(gal) & PVGA(gal) & PPHA(s) \\
\hline aic0160401061450 & 53.303 & 15.661 & 0.288 & myz0099610192344 & 31.052 & 16.220 & 0.8 \\
\hline aic0169703161451 & 109.096 & 29.596 & 0.152 & myz0099703261731 & 81.092 & 71.245 & 0.917 \\
\hline akt0150305261824 & 47.110 & 20.162 & 2.41 & myz0139610192344 & 229.640 & 84.696 & 0.5 \\
\hline akt0170305261824 & 70.175 & 34.327 & 2.41 & myz0139612030718 & 208.279 & 72.561 & 0.8 \\
\hline aom0110305261824 & 101.173 & 56.504 & 0.255 & myz0179610181950 & 55.295 & 12.236 & 0.513 \\
\hline ehm0150103241528 & 311.621 & 166.419 & 0.775 & myz0179610192344 & 216.474 & 40.146 & 0.602 \\
\hline fki0060006050954 & 65.57 & 71.989 & 0.585 & myz0179705131438 & 36.761 & 6.240 & 0.476 \\
\hline hkd0670104270249 & 174.328 & 60.037 & 0.226 & nig0250410231803 & 53.139 & 14.631 & 0.295 \\
\hline hkd0919905130259 & 243.218 & 85.732 & 0.362 & oit0100103241528 & 65.123 & 22.577 & 1.261 \\
\hline hkd1090309260450 & 240.714 & 84.400 & 0.302 & oit0109610192344 & 40.084 & 16.672 & 1.05 \\
\hline hkd1260309260450 & 189.484 & 54.113 & 1.366 & oit0160103241528 & 45.879 & 16.299 & 0.741 \\
\hline hkd1269905130259 & 33.028 & 8.348 & 0.336 & oit0160211041336 & 65.474 & 26.161 & 0.909 \\
\hline hrs0119805230449 & 11.700 & 3.868 & 2.924 & oit0169610192344 & 49.889 & 13.950 & 0.685 \\
\hline hyg0020101120800 & 818.023 & 33.648 & 0.909 & oky0100312131232 & 57.985 & 16.801 & 0.746 \\
\hline hyg0200409052357 & 21.382 & 7.942 & 1.107 & osk0060409051907 & 46.709 & 14.602 & 1.037 \\
\hline isk0060006070616 & 192.663 & 52.331 & 0.171 & sit0029612211029 & 95.010 & 34.219 & 0.273 \\
\hline iwt0030307260713 & 48.138 & 13.588 & 0.588 & szo0029703072135 & 231.317 & 72.902 & 0.383 \\
\hline iwt0200307260713 & 54.782 & 19.771 & 0.662 & szo0029703100009 & 56.319 & 22.100 & 0.347 \\
\hline iwt0200408101513 & 61.549 & 22.121 & 0.685 & szo0160007151030 & 20.995 & 5.593 & 0.73 \\
\hline iwt0209612222353 & 4.507 & 2.530 & 0.595 & szo0169703161451 & 45.139 & 7.005 & 0.202 \\
\hline kgs0040304121328 & 92.848 & 28.926 & 0.595 & tky0100007011602 & 197.242 & 125.969 & 1.05 \\
\hline kgs0049704030433 & 112.420 & 70.232 & 0.481 & tky0100007030503 & 107.974 & 55.508 & 0.441 \\
\hline kgs0049704092320 & 58.847 & 22.747 & 0.578 & tky0100007051121 & 50.120 & 22.760 & 0.476 \\
\hline kgs0049705131438 & 155.806 & 99.673 & 0.61 & tky0100007271049 & 180.647 & 114.433 & 0.719 \\
\hline kgs0079703261731 & 224.397 & 110.968 & 1.107 & tky0109611170052 & 88.926 & 43.829 & 0.481 \\
\hline kgs0079704030433 & 179.124 & 50.382 & 0.746 & tky0110007302125 & 195.409 & 111.054 & 0.153 \\
\hline kgs0079704051324 & 92.820 & 42.360 & 0.157 & ttr0080010061452 & 61.290 & 32.836 & 0.87 \\
\hline kgs0079705131438 & 317.534 & 149.385 & 0.719 & ttr0080010082051 & 98.513 & 93.088 & 0.952 \\
\hline kgs0109703261731 & 109.501 & 73.011 & 0.971 & ttr0080012190618 & 108.370 & 37.577 & 0.317 \\
\hline kgs0109704030433 & 66.082 & 32.182 & 0.8 & ttr0080209161010 & 28.264 & 12.103 & 0.247 \\
\hline kgs0109705131438 & 205.619 & 113.991 & 0.418 & ttr0089709040516 & 68.772 & 29.061 & 0.431 \\
\hline kgs0309701180037 & 25.341 & 9.897 & 0.481 & tym0020006070616 & 68.806 & 16.976 & 0.148 \\
\hline kgs0309701180053 & 51.234 & 21.626 & 0.518 & wky0070409051907 & 71.064 & 47.848 & 2.41 \\
\hline kmm0080006080932 & 235.797 & 94.671 & 0.175 & ymt0010305261824 & 22.185 & 15.229 & 2.558 \\
\hline koc0140104252340 & 44.200 & 18.213 & 0.909 & ymt0019902261418 & 51.911 & 14.686 & 0.481 \\
\hline mie0140409052357 & 133.824 & 47.294 & 0.685 & ymt0110305261824 & 45.862 & 22.510 & 0.719 \\
\hline myg0050305261824 & 179.971 & 111.990 & 0.102 & El Centro & 306.740 & 200.900 & 0.546 \\
\hline myg0050307260713 & 86.101 & 39.936 & 0.107 & NihonkaiChubu(Hachi) & 229.65 & 126.308 & 2.558 \\
\hline myg0059608110354 & 304.912 & 190.410 & 0.093 & taft & 152.7 & 106.82 & 0.36 \\
\hline myg0060307260713 & 146.404 & 100.260 & 1.261 & Miyagikenoki & 258.1 & 116.145 & 0.971 \\
\hline
\end{tabular}




\section{Tomoyo Taniguchi}

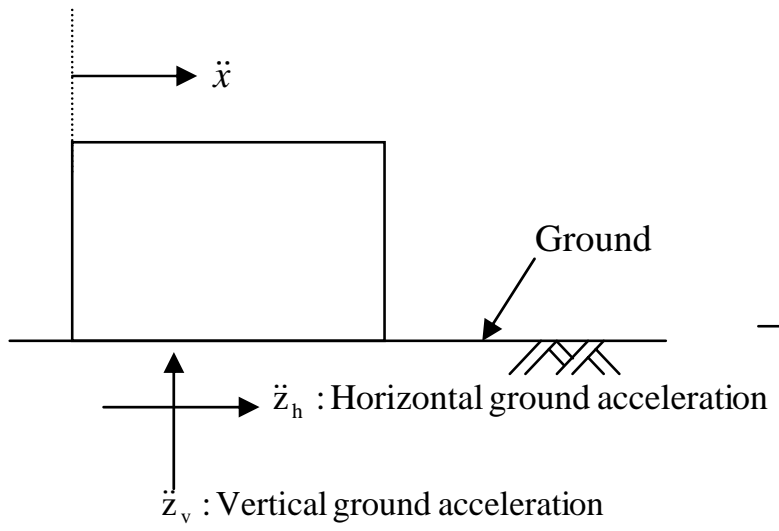

(a) The body on the ground

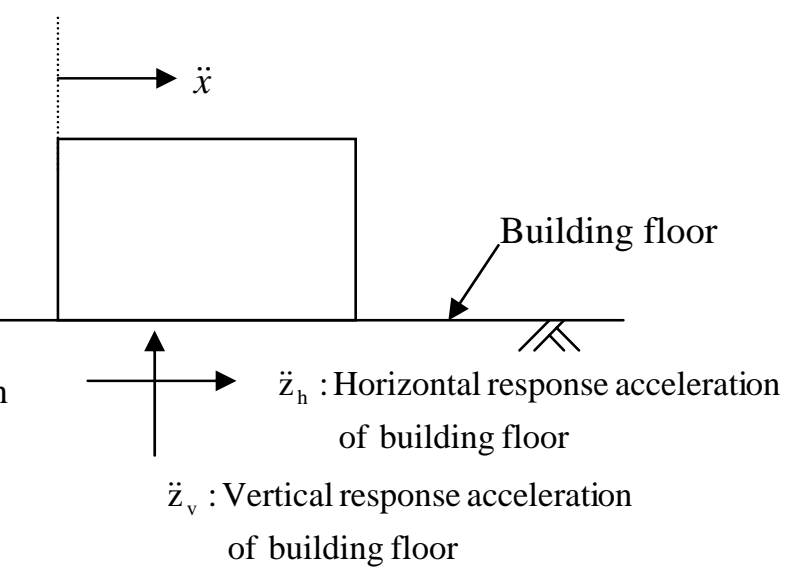

(b) The body on the building floor

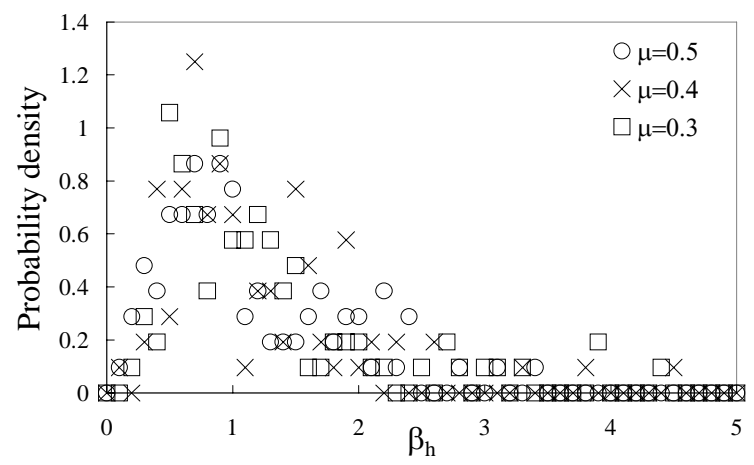

(c) Effects of vertical ground motion on the slip displacement (In case of PHGA=9m/ $\mathrm{s}^{2}$ )

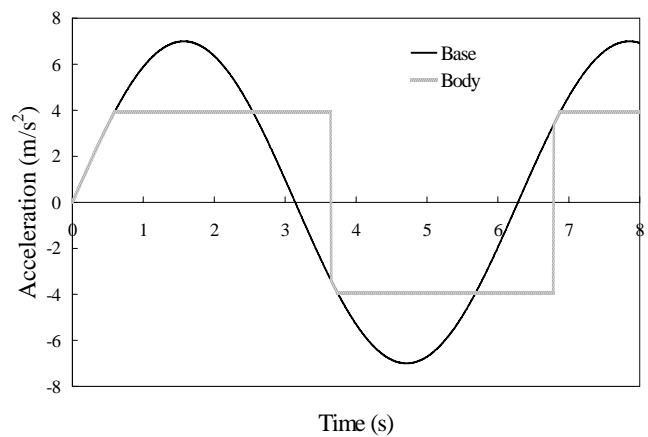

(d) Acceleration of base and body during slip

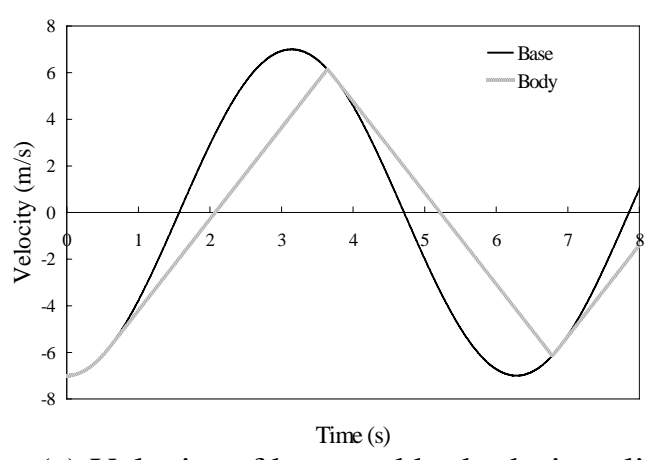

(e) Velocity of base and body during slip

Figure 1. Analytical models and slip motion of rigid body 
Tomoyo Taniguchi

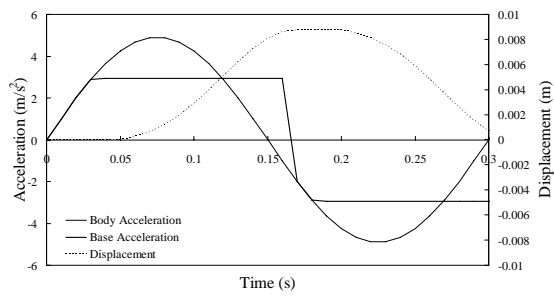

(a) $\left(A_{g x}, T, \mu\right)=(0.5,0.3,0.3)$

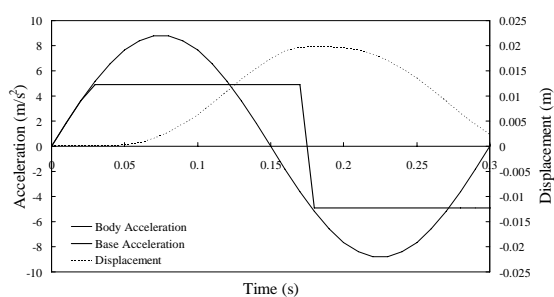

(d) $\left(A_{g x}, T, \mu\right)=(0.9,0.3,0.5)$

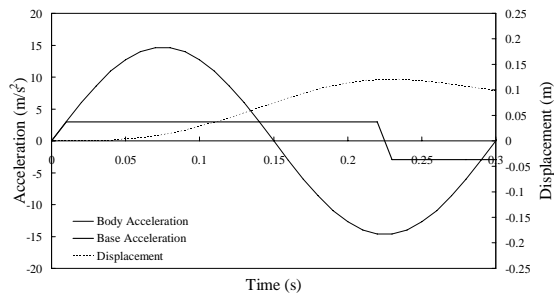

(g) $\left(A_{g x}, T, \mu\right)=(1.5,0.3,0.3)$



(b) $\left(A_{g x}, T, \mu\right)=(0.5,1.0,0.3)$

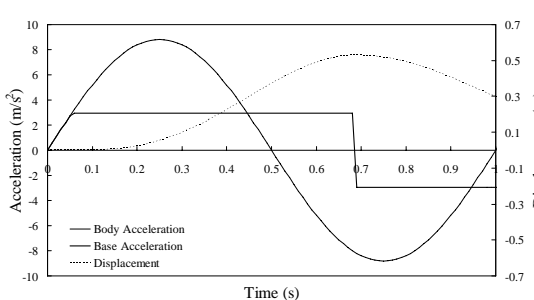

(e) $\left(A_{g x}, T, \mu\right)=(0.9,1.0,0.3)$

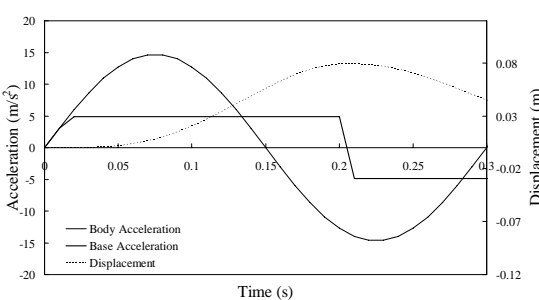

(h) $\left(A_{g x}, T, \mu\right)=(1.5,0.3,0.5)$

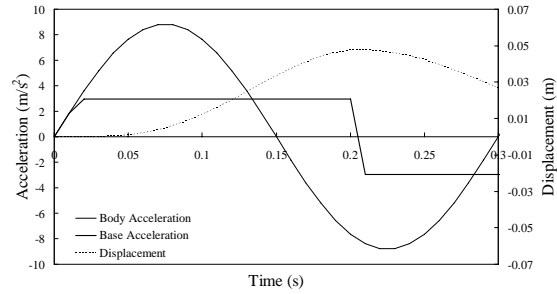

(c) $\left(A_{g x}, T, \mu\right)=(0.9,0.3,0.3)$

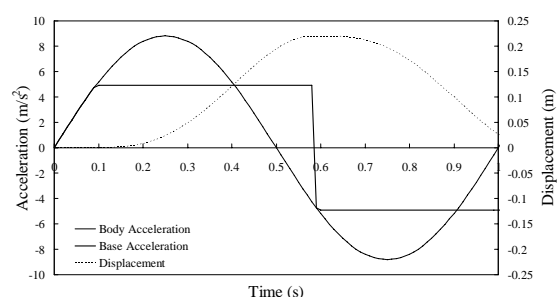

(f) $\left(A_{g x}, T, \mu\right)=(0.9,1.0,0.5)$

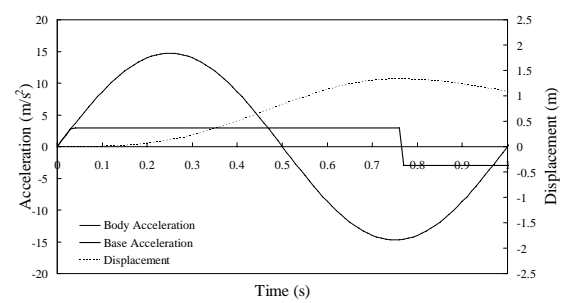

(i) $\left(A_{g x}, T, \mu\right)=(1.5,1.0,0.3)$

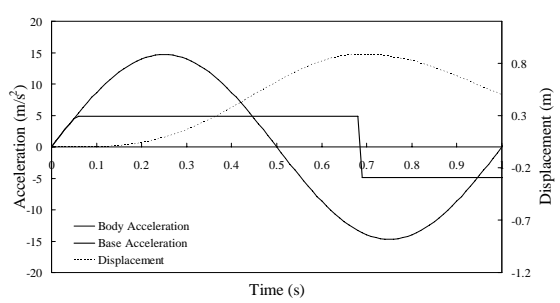

(j) $\left(A_{g x}, T, \mu\right)=(1.5,1.0,0.5)$

\begin{tabular}{|r|c|c|r|r|c|}
\hline$A_{g x}$ & $T$ & $\mu$ & \multicolumn{1}{|c|}{ Eq.(15) } & \multicolumn{1}{c|}{ Exact disp. } & Error \\
\hline 0.5 & 0.3 & 0.3 & $-0.00881 \mathrm{~m}$ & $-0.00875 \mathrm{~m}$ & $0.612 \%$ \\
\hline 0.5 & 1.0 & 0.3 & $-0.0979 \mathrm{~m}$ & $-0.0978 \mathrm{~m}$ & $0.067 \%$ \\
\hline 0.9 & 0.3 & 0.3 & $-0.0476 \mathrm{~m}$ & $-0.0476 \mathrm{~m}$ & $0.039 \%$ \\
\hline 0.9 & 0.3 & 0.5 & $-0.0198 \mathrm{~m}$ & $-0.0198 \mathrm{~m}$ & $0.110 \%$ \\
\hline 0.9 & 1.0 & 0.3 & $-0.529 \mathrm{~m}$ & $-0.529 \mathrm{~m}$ & $0.061 \%$ \\
\hline 0.9 & 1.0 & 0.5 & $-0.220 \mathrm{~m}$ & $-0.220 \mathrm{~m}$ & $0.001 \%$ \\
\hline 1.5 & 0.3 & 0.3 & $-0.120 \mathrm{~m}$ & $-0.120 \mathrm{~m}$ & $0.380 \%$ \\
\hline 1.5 & 0.3 & 0.5 & $-0.0794 \mathrm{~m}$ & $-0.0794 \mathrm{~m}$ & $0.003 \%$ \\
\hline 1.5 & 1.0 & 0.3 & $-1.33 \mathrm{~m}$ & $-1.33 \mathrm{~m}$ & $0.404 \%$ \\
\hline 1.5 & 1.0 & 0.5 & $-0.882 \mathrm{~m}$ & $-0.882 \mathrm{~m}$ & $0.067 \%$ \\
\hline
\end{tabular}

(k) Comparison of the results of Eq. (15) with numerical results

Figure 2. Prediction accuracy of proposed method 
Tomoyo Taniguchi

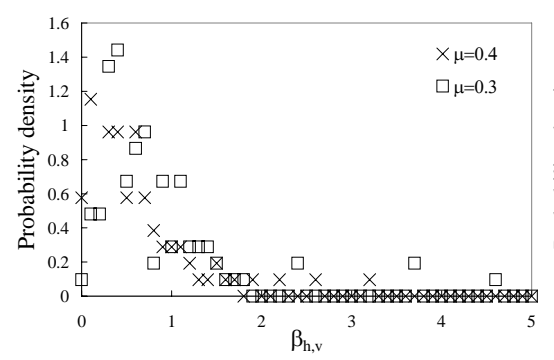

(a) $P H G A=5 m / s^{2}$

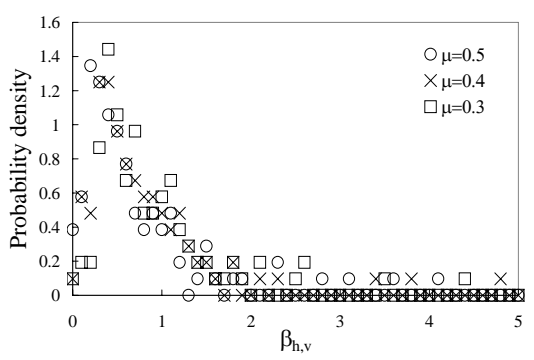

(b) PHGA $=7 \mathrm{~m} / \mathrm{s}^{2}$

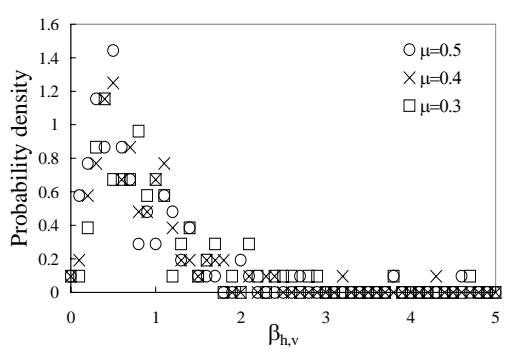

(c) PHGA $=9 \mathrm{~m} / \mathrm{s}^{2}$

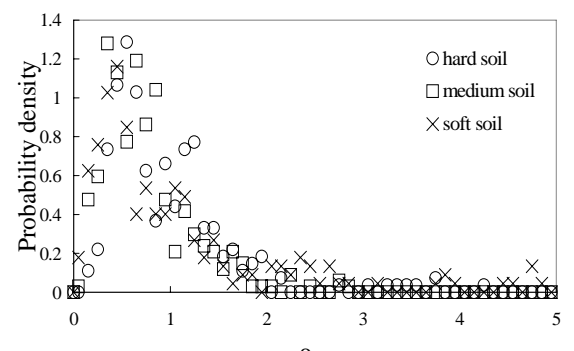

$\beta_{\mathrm{h}, \mathrm{v}}$

(d) Classification according to soil types

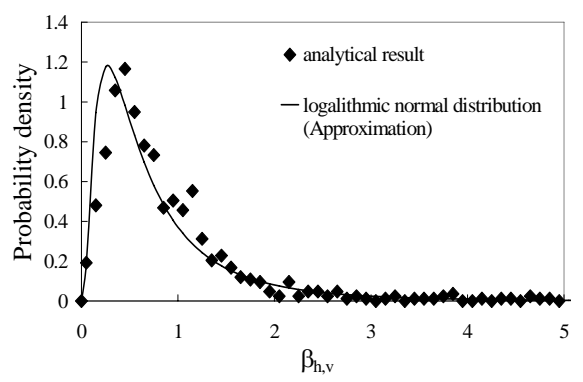

(e) All results

Figure 3. Probability density of slip ratio $\beta_{h, v}$ 
Tomoyo Taniguchi

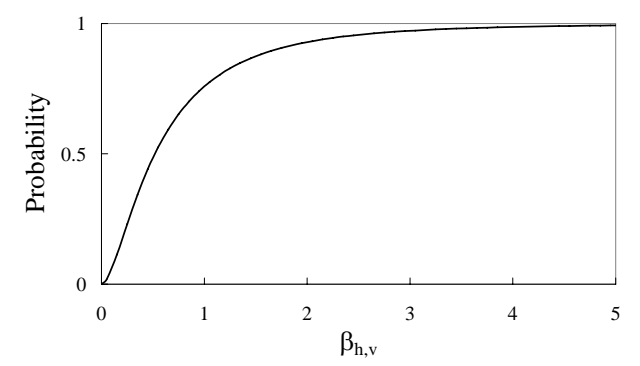

\begin{tabular}{|c|c|}
\hline$\beta_{h, v}^{\text {Prob }}$ & Probability of nonexceedence \\
\hline 1.69 & $90 \%$ \\
\hline 2.35 & $95 \%$ \\
\hline 2.90 & $97 \%$ \\
\hline 4.40 & $99 \%$ \\
\hline
\end{tabular}

Figure 4. Probability of slip ratio $\beta_{h, v}$ and modification factor $\beta_{h, v}^{\text {Prob }}$ for selected probability of nonexceedance 


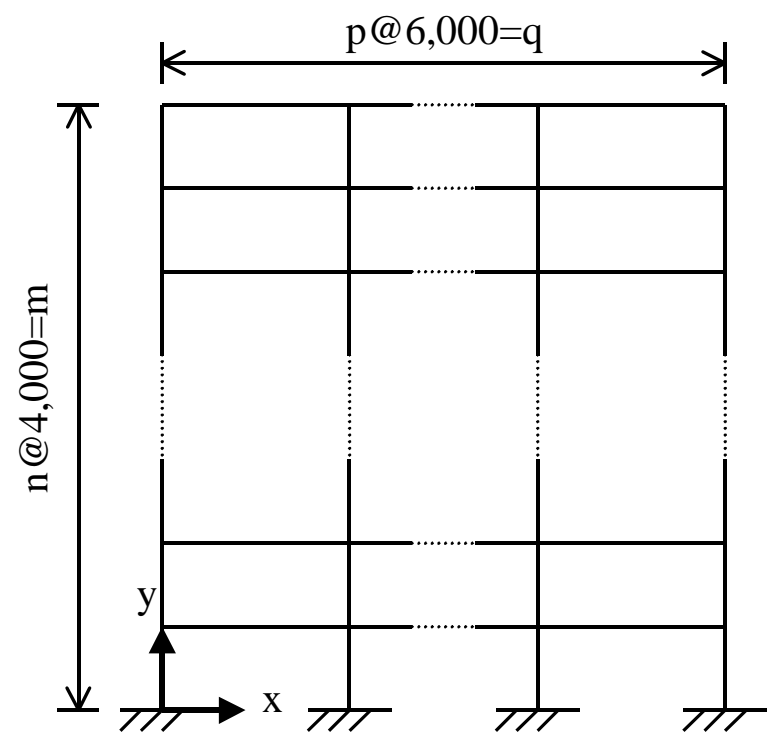

unit[mm]

\begin{tabular}{|c|c|c|c|c|}
\hline Model & $\mathrm{n}$ & $\mathrm{m}(\mathrm{mm})$ & $\mathrm{p}$ & $\mathrm{q}(\mathrm{mm})$ \\
\hline 5-story & 5 & 20,000 & 2 & 12,000 \\
\hline 10-story & 10 & 40,000 & 3 & 18,000 \\
\hline 20-story & 20 & 120,000 & 4 & 24,000 \\
\hline
\end{tabular}

(a) Configuration of multi-story structure model

\begin{tabular}{|c|c|c|c|}
\hline Model & Story & Column Section & Beam Section \\
\hline \multirow{2}{*}{ 5-story } & $1-3$ & $650 \times 650$ & $450 \times 600$ \\
\cline { 2 - 4 } & $4-5$ & $600 \times 600$ & $400 \times 600$ \\
\hline \multirow{3}{*}{ 10-story } & $1-3$ & $800 \times 800$ & $450 \times 850$ \\
\cline { 2 - 4 } & $4-7$ & $750 \times 750$ & $400 \times 800$ \\
\cline { 2 - 4 } & $8-10$ & $650 \times 650$ & $350 \times 700$ \\
\hline \multirow{4}{*}{ 20-story } & $1-5$ & $850 \times 850$ & $600 \times 1000$ \\
\cline { 2 - 4 } & $6-10$ & $800 \times 800$ & $600 \times 800$ \\
\cline { 2 - 4 } & $11-15$ & $750 \times 750$ & $550 \times 750$ \\
\cline { 2 - 4 } & $16-20$ & $650 \times 650$ & $500 \times 700$ \\
\hline
\end{tabular}

(b) Dimensions of member (Unit: $\mathrm{mm}$ )

\begin{tabular}{|c|c|c|c|}
\hline Model & 1st mode & 2nd mode & 3rd mode \\
\hline 5-story & 0.466 & 0.152 & 0.082 \\
\hline 10-story & 0.834 & 0.308 & 0.183 \\
\hline 20-story & 1.706 & 0.630 & 0.375 \\
\hline
\end{tabular}

(c) Natural period of each mode (Unit: s)

Figure 5. Building model 
Tomoyo Taniguchi

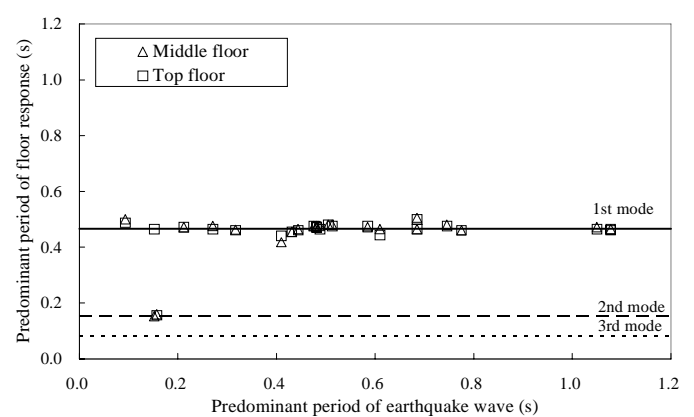

(a) observed period (5-story, linear bldg)

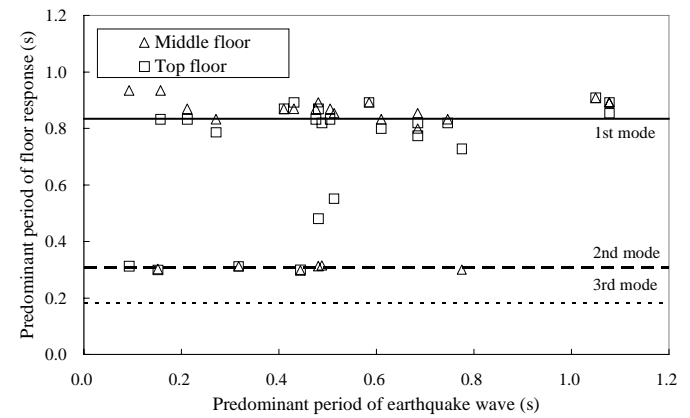

(b) observed period (10-story, linear bldg)

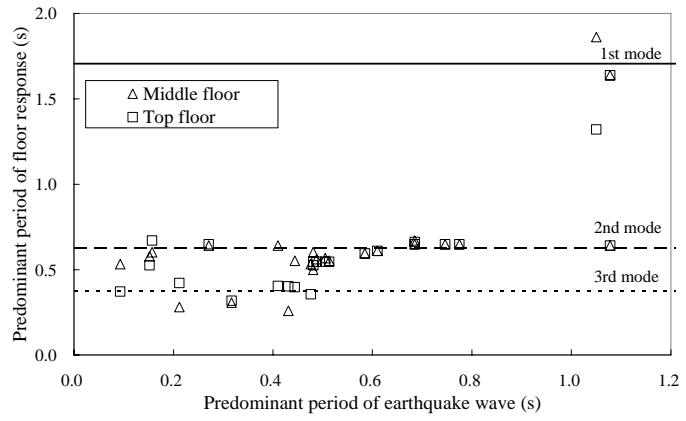

(c) observed period (10-story, linear bldg)

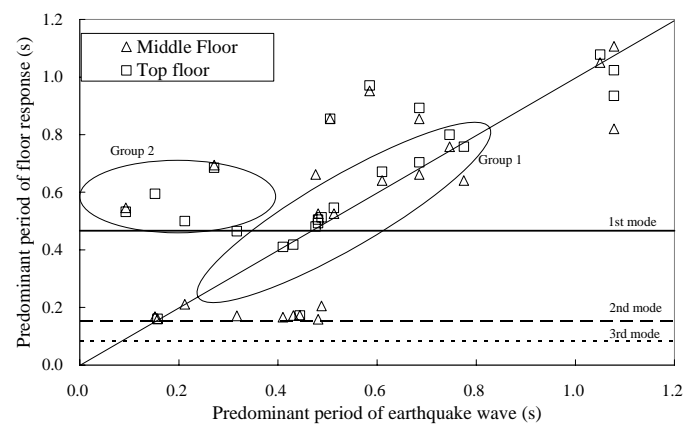

(d) observed period (5-story, nonlinear bldg)

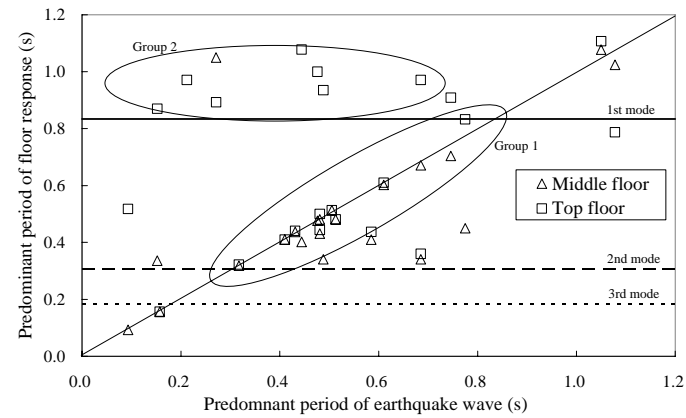

(e) observed period (10-story, nonlinear bldg)

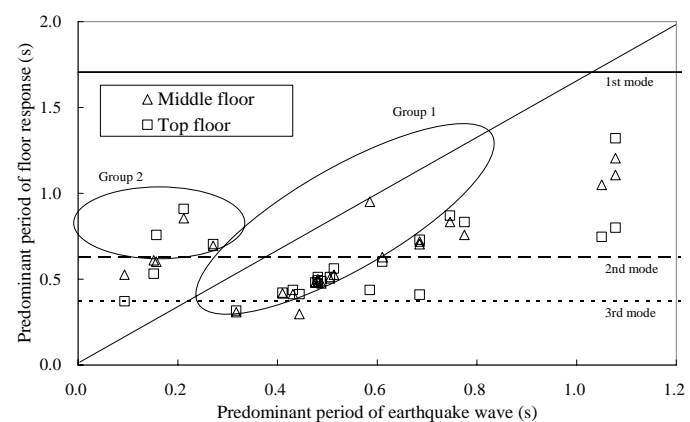

(f) observed period (20-story, nonlinear bldg)

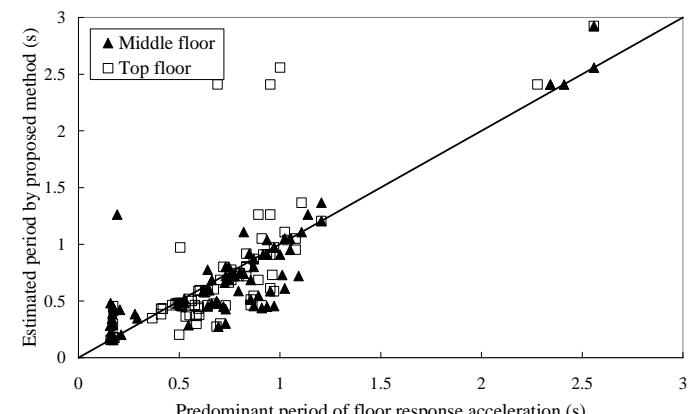

(g) accuracy of proposed method (5-story all case)

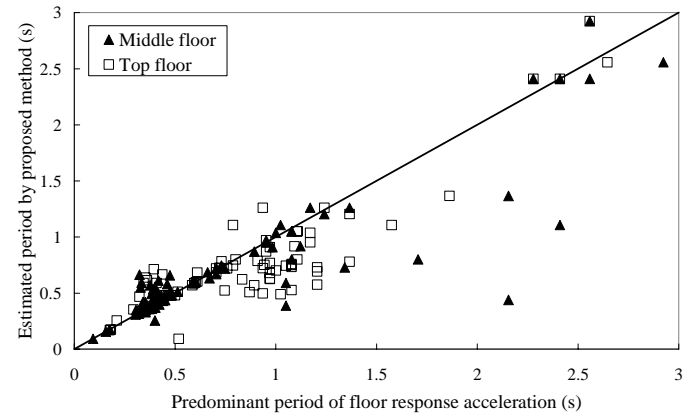

(h) accuracy of proposed method (10-story all case)

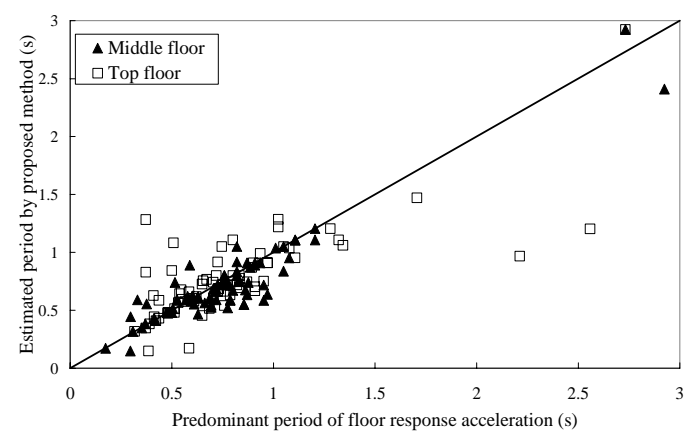

(i) accuracy of proposed method (20-story all case)

Figure 6. Predominant period of HFRA and its estimation accuracy 
Tomoyo Taniguchi

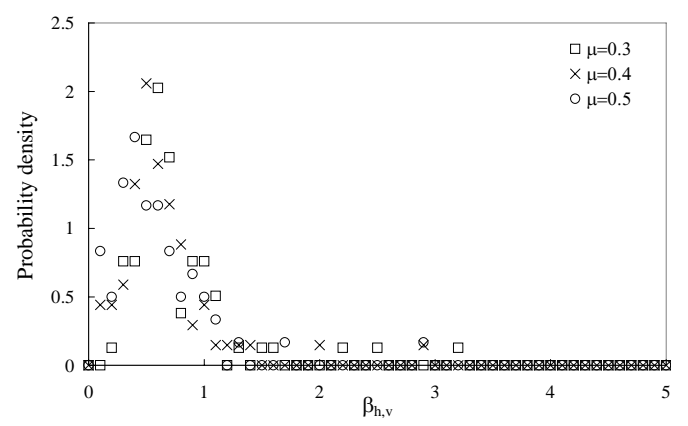

(a) Middle floor on five-story building

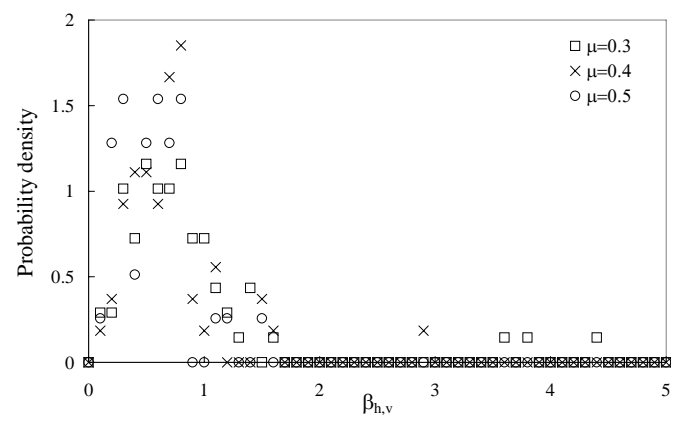

(c) Middle floor on ten-story building

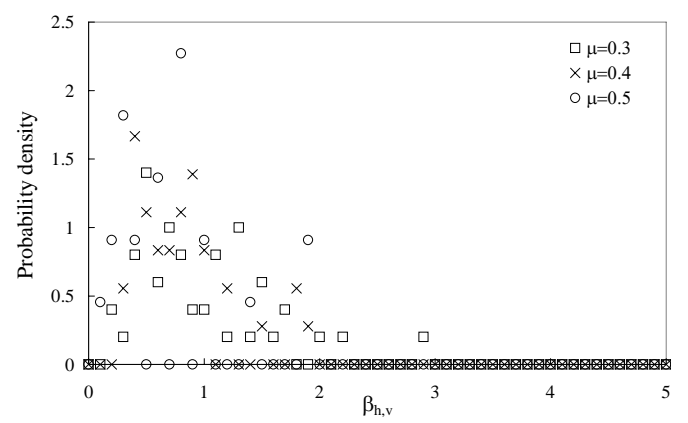

(e) Middle floor on twenty-story building

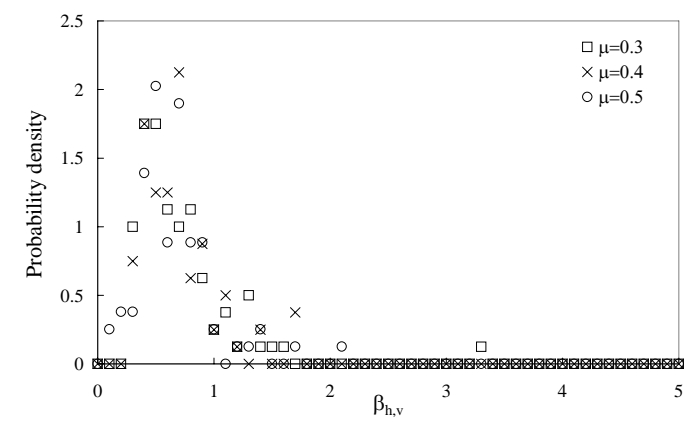

(b) Top floor on five-story building

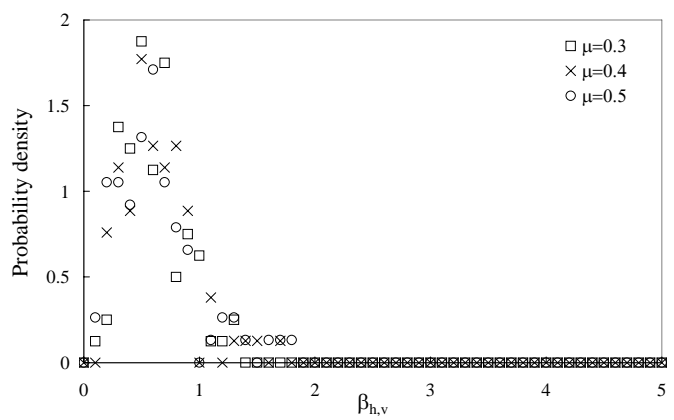

(d) Top floor on ten-story building

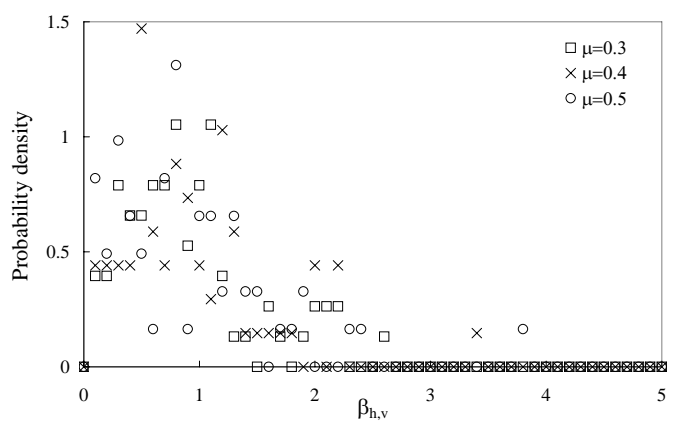

(f) Top floor on twenty-story building



(g) All results

Figure 7. Probability density of slip ratio $\beta_{h, v}$

(Buildings in linear state) 


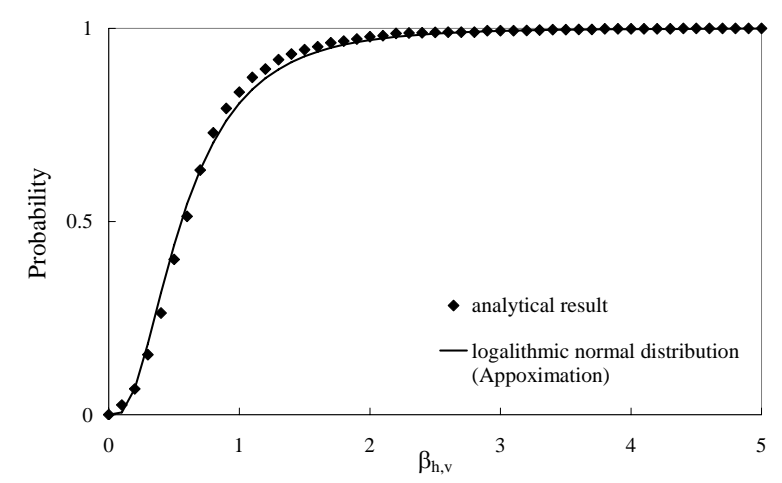

\begin{tabular}{|c|c|}
\hline$\beta_{h, v}^{\text {Prob }}$ & Probability of nonexceedence \\
\hline 1.33 & $90 \%$ \\
\hline 1.71 & $95 \%$ \\
\hline 2.01 & $97 \%$ \\
\hline 2.72 & $99 \%$ \\
\hline
\end{tabular}

Figure 8. Probability of slip ratio $\beta_{h, v}$ and modification factor $\beta_{h, v}^{\text {Prob }}$ for selected probability of nonexceedance

(Buildings in linear state) 
Tomoyo Taniguchi

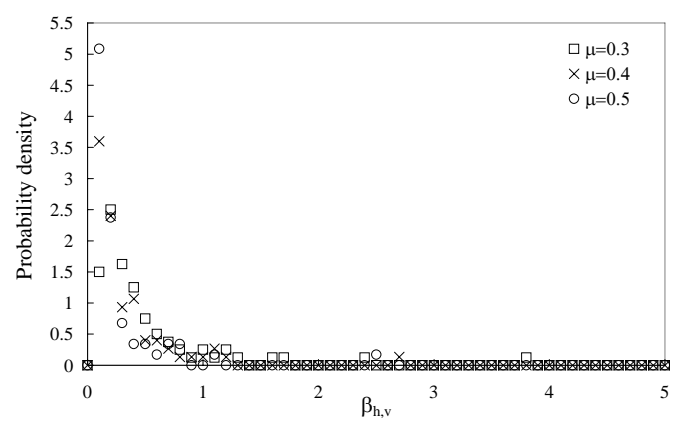

(a) Middle floor on five-story building

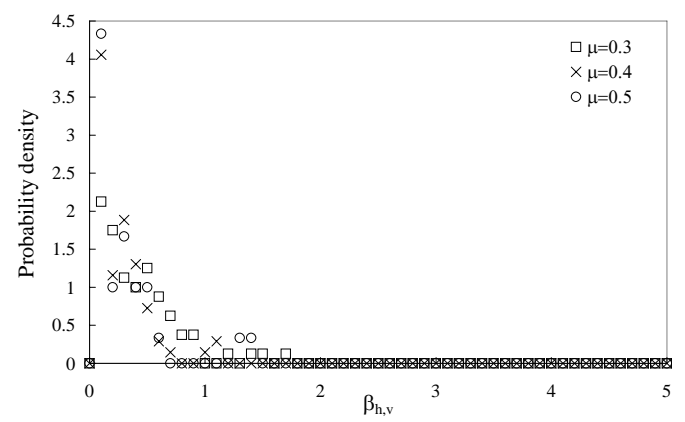

(c) Middle floor on ten-story building

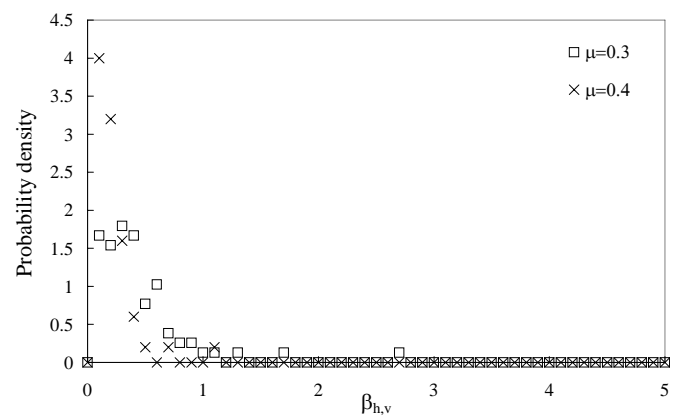

(e) Middle floor on twenty-story building

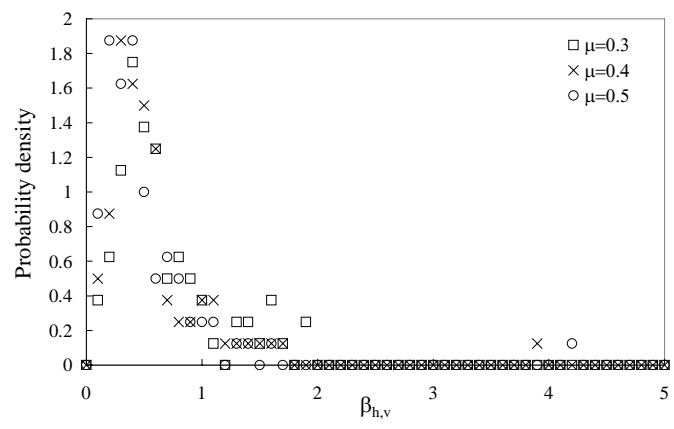

(b) Top floor on five-story building

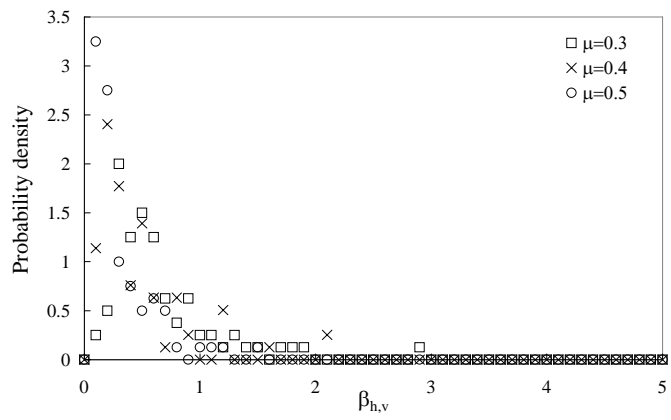

(d) Top floor on ten-story building

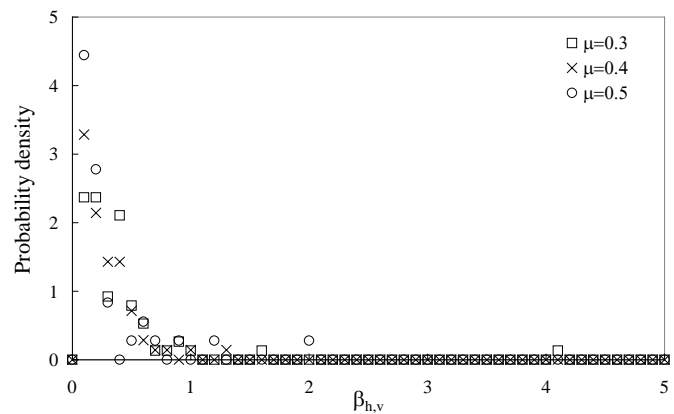

(f) Top floor on twenty-story building

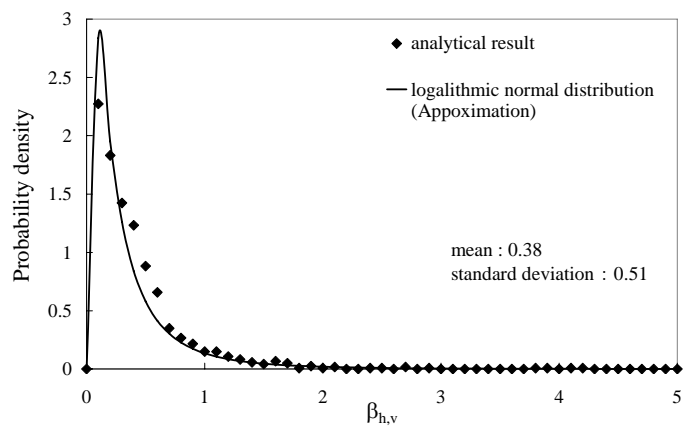

(g) All results

Figure 9. Probability density of slip ratio $\beta_{h, v}$

(Buildings in nonlinear state) 


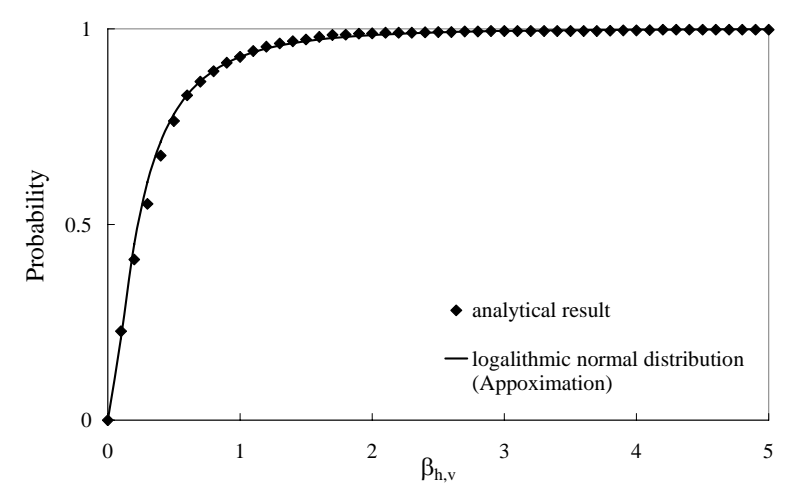

\begin{tabular}{|c|c|}
\hline$\beta_{h, v}^{\text {Prob }}$ & Probability of nonexceedence \\
\hline 0.84 & $90 \%$ \\
\hline 1.21 & $95 \%$ \\
\hline 1.54 & $97 \%$ \\
\hline 2.41 & $99 \%$ \\
\hline
\end{tabular}

Figure 10. Probability of slip ratio $\beta_{h, v}$ and modification factor $\beta_{h, v}^{\text {Prob }}$ for selected probability of nonexceedance

(Buildings in nonlinear state) 
Tomoyo Taniguchi

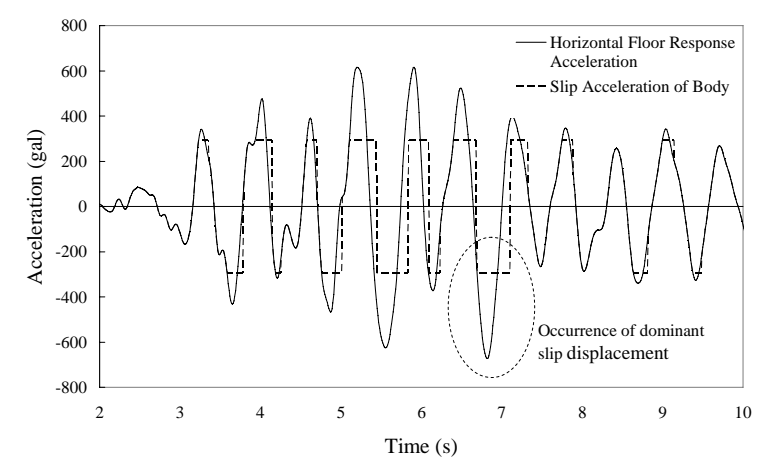

(a) HFRA and slip acceleration (linear bldg)

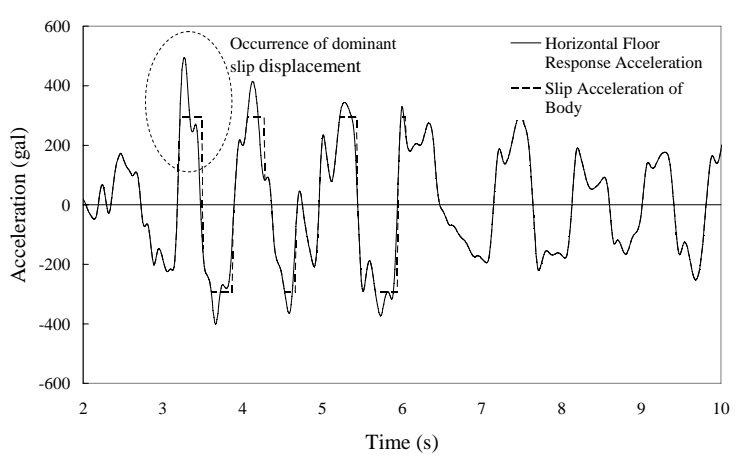

(c) HFRA and slip acceleration (nonlinear bldg)

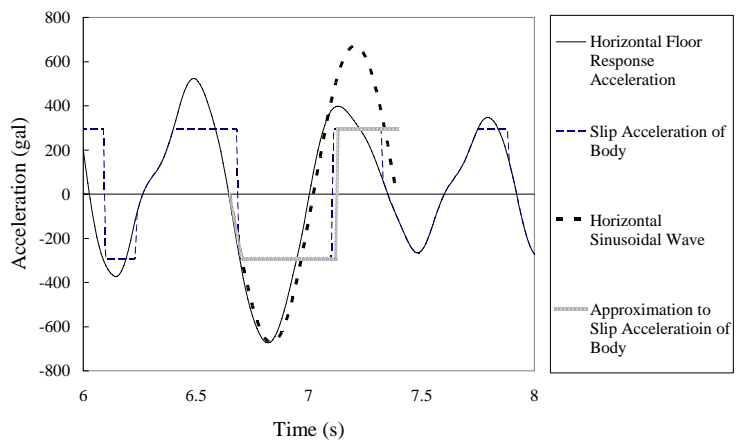

(b) Magnifying view of the onset of dominant slip (linear bldg)

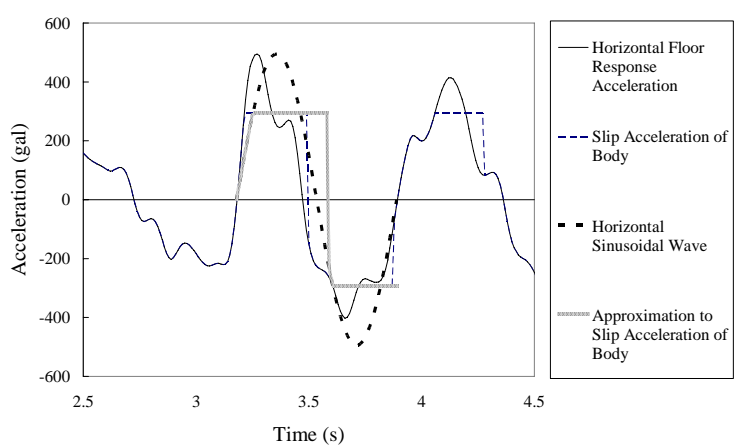

(d) Magnifying view of the onset of dominant slip (nonlinear bldg)

Figure 11. Comparison of HFRA at linear/nonlinear building to horizontal sinusoidal acceleration and slip acceleration induced by them 